“드 2014 IEEE. Personal use of this material is permitted. Permission from IEEE must be obtained for all other uses, in any current or future media, including reprinting/republishing this material for advertising or promotional purposes, creating new collective works, for resale or redistribution to servers or lists, or reuse of any copyrighted component of this work in other works." 


\title{
Analysis of the influence to productivity of software corrective maintenance using an economic model
}

\author{
Kai Huang, Xiaoying Kong, Kumbesan Sandrasegaran \\ Faculty of Engineering and Information Technology \\ University of Technology, Sydney, Australia
}

\begin{abstract}
This paper presented an economic model for productivity of software corrective maintenance. The productivity is modeled using economic value of the maintenance process as the output, and the pre-committed fixed cost and variable cost as input. The relationship of the economic value and these cost components are modeled using analytical theory of investment. The values of corrective maintenance process are analyzed. A simulation approach is demonstrated to analyze the influences to the productivity in corrective maintenance. This approach provided a tool to identify and analyze the optimal parameters in productivity using the economic model and simulation.
\end{abstract}

Keywords- Economic model, Simulation, Software maintenance, Corrective maintenance, Influence factor

\section{INTRODUCTION}

Software development lifecycle consists of a number of phases: planning, requirements, specifications, design, implementation, integration, testing, deployment, operation and maintenance. After new development is completed and software system is delivered to client for use, the life cycle goes to a long maintenance phase. IEEE standard defines software maintenance as "the process of modifying a software system or component after delivery to correct faults, improve performance or other attributes, or adapt to a changed environment" [1]. Research found that the main efforts in a software development life cycle have been made in maintenance phase [2,3]. Software project managers' viewpoint of activity importance is more in maintenance phase than in new software development [4].

To understand software development efforts, large amount of research work have been focusing on cost models for new software development. Conventional approaches include Constructive Cost Model (COCOMO) [5] and functional points [6]. Recently developed dynamic model includes an economic model using analytical theory of project investment [7]. These models are developed for early new development phases in life cycle rather than maintenance. The characteristics of maintenance activity are different from new software development [4,8]. Rather than straight forward creation of new software, maintenance activities make changes to existing software system in a long period in a relatively stable environment. There are three major maintenance types: corrective maintenance, adaptive maintenance and perfective maintenance $[1,8]$. Therefore the economic model for maintenance phase should be separated from new development phase. In literatures, Some researcher attempt to develop models to analyse maintenance workload, cost and productivity. Examples of such models are Jorgensen's productivity model for maintenance activity [9], Basili's productivity using metric of line of code per hour [10], Nguyen's maintenance metrics using maintenance size, effort and effort distribution [2], and COCOMO maintenance model [5]. These models are static models using metrics.

In this research, we develop a new dynamic economic model to analyse software maintenance productivity using analytical modelling and simulation approach. Maintenance efforts and values will be analysed using this economic model. There are three types of maintenance: corrective maintenance, adaptive maintenance and perfective maintenance. In this paper, we will analyse the productivity model in corrective maintenance type. Impact factors to corrective maintenance productivity will be analysed using simulation.

This paper is organized as follows. Section II will review related work in software maintenance modelling. Section III will present the new analytical economic model of software maintenance productivity for corrective maintenance. Section IV will present simulation results using the economic model for corrective maintenance. Selected impact factors are analysed. Conclusion and further research will be drawn in Section V.

\section{SOFTWARE MAINTENANCE PRODUCTIVITY AND INFLUENCE FACTORS}

To model and evaluate the software development efforts, productivity is used as one of the major metrics. Productivity is defined as "the ratio of work product to work effort" by IEEE standard [11]. Software productivity can be measured by the ratio of "an output primitive" to "its corresponding primitive" of the software [11]. Software maintenance productivity could be similarly defined. In this section we will review the related work on software maintenance productivity.

Software maintenance productivity can be measured using maintenance production function. Maintenance production function represents the relationship between the maintenance effort (major maintenance input) and modified software (major maintenance output). In literature, the metrics for maintenance productivity have been designed in different approaches. Productivities in different maintenance types are different. Maintenance activities are classified into three major types: corrective, adaptive and perfective maintenance $[2,8]$. Some literature has modelled maintenance productivity in general or in different types. 
In Jorgensen's approach, maintenance productivity is defined using the ratio of maintenance size to effort used on the maintenance task [9]. The maintenance size is based on inserted line of code (LOC), updated LOC and deleted LOC in a maintenance task. Boehm uses the average Delivered Source Instruction (DSI) of a Man in a Month (MM) to define the productivity metric [5]. In Basili's model, software maintenance productivity is defined using Source Lines Of Code (SLOC) over hours spent in the maintenance task [10]. They also found that the productivity measurements of different types of maintenance activities are different [10]. The productivity for error correction activity and mixed activity was significantly lower than for enhancement activity, implying that error corrections were less productive-in terms of SLOC per hour-than in enhancements of perfective maintenance type [10]. Nguyen et al. continued Basili's study. They assessed the maintenance size, effort and effort distributions of three different maintenance types - corrective, adaptive, perfective maintenance, then built estimation models to predict the developers' effort spent on maintenance tasks [2]. Their results indicated that corrective maintenance was the least productive maintenance in all maintenance types. Half effort of maintenance was spent on reading and understanding present code.

Recently an analytical modeling approach is proposed to model software productivity in general using economic values and software economic cost [7]. The software productivity metric is defined using the ratio of total software project value and total pre-committed project fixed cost pluses total variable cost of the project. Eq. (1) represents the metric of productivity in this analytical approach.

$$
\text { Productivity_Eco }=\frac{S_{-} \text {Total }}{K+C_{-} \text {Total }}
$$

where Productivity_Eco is the productivity metric of a software project. $S_{-}$Total is the total software project economic values. $K$ is the total pre-committed project fixed cost $C_{-}$Total is the total variable cost in the software project.

Using this analytical model, the impact factors to the software project can be identified and analysed using simulation [7]. This analytical model is developed for new software development. Characteristics of maintenance phase are not analysed.

In our research, we will use this dynamic analytical modelling approach to analyse the productivity for software maintenance.

In these literatures, some influences to maintenance process are identified. By analysing these influences in these literatures, we derived the influence factors to maintenance productivity and classify them into two groups: technical factors and non-technical factors. These influence factors and groups are listed in Table 1.

TABLE 1 FACTORS AFFECTING SOFTWARE MAINTENANCE PRODUCTIVITY

\begin{tabular}{|c|l|}
\hline Group & \multicolumn{1}{|c|}{ Influence Factors } \\
\hline \multirow{2}{*}{$\begin{array}{c}\text { Technical } \\
\text { factors }\end{array}$} & Quality of system original program \\
\cline { 2 - 2 } & Code reusability \\
\cline { 2 - 2 } & Modern programming practice \\
\hline
\end{tabular}

\begin{tabular}{|l|l|}
\hline \multirow{5}{*}{} & Quality of system documentation \\
\cline { 2 - 2 } & Adequacy of system design specification \\
\cline { 2 - 2 } & Maintenance personnel experience \\
\cline { 2 - 2 } & Maintenance personnel abilities on maintenance \\
\cline { 2 - 2 } & $\begin{array}{l}\text { Maintenance personnel abilities on configuration } \\
\text { management }\end{array}$ \\
\cline { 2 - 2 } & Maintenance tool availability \\
\cline { 2 - 2 } & Database size \\
\cline { 2 - 2 } & Software system complexity \\
\hline \multirow{5}{*}{ Non-technical factors } & User demands for enhancements, extents \\
\cline { 2 - 2 } & Software reliability request \\
\cline { 2 - 2 } & Usage time \\
\cline { 2 - 2 } & Maintenance personnel stability \\
\cline { 2 - 2 } & Environmental change \\
\hline
\end{tabular}

We will use these factors to analyse the productivity in this research.

\section{ECONOMIC MODEL FOR SOFTWARE MAINTENANCE}

In this section, we present an economic model for software maintenance process. Productivity of maintenance is modelled. In this model, software maintenance productivity is defined using the output values and input cost from economic viewpoint.

\section{A. Software Maintenace Economic Model}

To define a metric for software maintenance productivity, we can use the ratio of the output and input of a maintenance process. From economic perspective, in software maintenance, the output is the economic value of the maintenance process, and the output is the total maintenance cost. The cost structure of a software project can be divided into pre-committed fixed cost and variable cost. Fixed cost includes cost before maintenance commences, such as education cost for maintenance personnel. Variable cost is consumed for the maintenance activity, such as salary paid for maintenance personnel in the maintenance activity.

Using analytical theory of project investment, the productivity of a software maintenance process in general can be represented as [7, 12, 13]:

$$
\text { Productivity_Eco }=\frac{S_{M T_{-} \text {Total }}}{K_{M T}+C_{M T_{-} \text {Total }}}
$$

where Productivity_Eco is the metric of productivity of the software maintenance process, $S_{M T_{-} \text {Total }}$ is the total economic value of the maintenance process, $K_{M T}$ is the pre-committed fixed cost, $C_{M T_{-} \text {Total }}$ is the total variable cost of the maintenance. This definition can be applied to three types of maintenance.

If the unit economic value of the maintenance in a unit time is $S_{M T}$, the unit variable cost in a unit time is $C_{M T}$, and the total time of the maintenance is $T$, the total value and the total variable cost can be represented as

$$
S_{M T_{-} \text {Total }}=S_{M T} \times T ; \quad C_{M T_{-} \text {Total }}=C_{M T} \times T
$$

The relationship between unit value, unit variable cost, fixed cost and the maintenance time can be represented as: 


$$
C_{M T}=S_{M T} N\left(d_{1}\right)-K_{M T} e^{-r T} N\left(d_{2}\right)
$$

where $N(d 1)$ and $N(d 2)$ are the cumulative probability distribution functions for a standardized normal random variable. $d_{1}$ and $d_{2}$ are calculate as below:

$$
\begin{aligned}
& d_{1}=\frac{\ln \left(S_{M T} / K_{M T}\right)+\left(r+\sigma^{2} / 2\right) T}{\sigma \sqrt{T}} \\
& d_{2}=\frac{\ln \left(S_{M T} / K_{M T}\right)+\left(r-\sigma^{2} / 2\right) T}{\sigma \sqrt{T}}=d_{1}-\sigma \sqrt{T}
\end{aligned}
$$

where $r$ is the discount rate, $\sigma$ is the uncertainty level.

Eq. 2 with relationships in Eq.3, Eq. 4, and Eq. 5 are the economic model for maintenance productivity. This model is a general model for 3 types of maintenance. For each type of maintenance, the factors in Table 1 will have different influence to productivity.

IEEE standards defined three maintenance types: corrective, adaptive and perfective maintenance. The input cost and output values are different in each type. Corrective maintenance is defined by IEEE standard as "the reactive modification of a software product performed after delivery to correct discovered problems [1]. Adaptive maintenance is the modification of a software product, performed after delivery, to keep a software product usable in a changed or changing environment [1]. Perfective maintenance is the modification of a software product after delivery to detect and correct latent faults in the software product before they are manifested as failures [1]. As a pilot study, in this paper, we focus on the corrective maintenance modelling. Economic models for adaptive and perfective maintenance will be analysed in further research.

\section{B. Analysis Productivity for Corrective Maintenace}

To evaluate the productivity in corrective maintenance, the economic value, the cost of the maintenance process, and the influence factors to the productivity will be analysed.

\section{1) Analysis of the value of corrective maintenance}

Corrective maintenance ensures the system run correctly and does not break down. Considering the system situation without any maintenance, the problem of the system will emerge and new similar systems will come to the market soon. This causes the intangible depreciation. The economic value of the software system will decrease rapidly without maintenance.

Suppose that the original value of the system at the time the development finishes is $S_{D e v}$. The value of the system depreciation of maintenance time $T$ can be assumed using an exponential accelerated model as below:

$$
S_{\text {No_ } M T}=S_{D e v} \times \alpha^{T}{ }_{N o_{-} M T} \quad 0<\alpha_{N o_{-} M T}<1
$$

where $S_{N o \_M T}$ is the unit value of the system in a unit time without maintenance. $\alpha_{N_{-} M T}$ is the depreciation rate without maintenance.

If corrective maintenance is carried out, the depreciation rate slows down. The lifetime of the system is extended.
Assuming the depreciation rate of the system with corrective maintenance is $\alpha_{C_{C r}-M T}$, the value of the system with maintenance of time $T$ is as below:

$$
S_{\text {WithCorr_MT }}=S_{\text {Dev }} \times \alpha^{T} \text { Corr }-M T_{1} \quad 0<\alpha_{\text {Corr }_{-} M T}<1
$$

where $S_{\text {WithCorr }}$ MT is the unit value of the system with maintenance, $\alpha_{\text {Cor_ }_{-} M T}$ is the depreciation rate with corrective maintenance. $\alpha_{N_{-} M T}<\alpha_{\mathrm{Corr}_{-} M T}$.

Therefore the value of the corrective maintenance $S_{M T}$ is:

$$
\begin{aligned}
S_{M T} & =S_{\text {WithCorr_MT }}-S_{N_{-} M T} \\
& =S_{D e v} \times\left(\alpha^{T} \text { Corr_MT }_{-}-\alpha^{T}{ }_{\text {No_MT }}\right)
\end{aligned}
$$

\section{2) Output: the cost of the corrective maintenance}

Usually purchasing new equipment and software is not needed in corrective maintenance process. The only cost is the cost of maintenance personnel. This cost is composed of two components: pre-committed fixed cost and variable cost.

\section{INFLUENCES TO CORRECTIVE MAINTENANCE}

To understand the influences to the productivity model, we use a simulation approach to analyse how the productivity changes with parameters in the productivity model, and how factors influence the productivity through those parameters. The parameters to be analysed in the model are: unit economic value of the maintenance in a unit time $\left(S_{M T}\right)$, pre-committed fixed cost of maintenance $\left(K_{M T}\right)$, variable cost of maintenance $\left(C_{M T}\right)$, system maintenance time $(T)$, discount rate of return $(r)$, and the rate of uncertainty $(\sigma)$.

\section{A. Basic economic productiviy model for software corrective mainteance}

Firstly we analyse the basic economic productivity without influences of factors. Keeping the values of $S_{D e v}, K, r, \sigma$, $\alpha_{C o r_{-} M T}$ and $\alpha_{N_{-} M T}$ unchanged, observe how $S_{M T}$ and the productivity change with the maintenance time $T$. In simulation, we set the value of the new developed system $S_{D e v}$ to $\$ 10,000$, fixed cost $K$ to $\$ 1,000$, discount rate $r$ at $3 \%$ per month, and uncertainty level 0.25 , depreciation rate with corrective maintenance $\alpha_{C_{\text {Crr }} M T} 0.8$, depreciation rate without maintenance $\alpha_{N_{-} M T} 0.5$. The system maintenance time $T$ changes from 2 months to 22 months with the interval of 2 months. Using the economic model in Eq. (2), (3), (4) and (5) for simulation, the changes of system maintenance value and the productivity without influence of factors are shown in Fig. 1 and Fig. 2. Simulation results in Fig 1 and Fig 2 demonstrate that without the influences of factors, the value of corrective maintenance decreases rapidly with the system depreciation. The productivity shows a curve of a reversed U. It rises from the beginning, bends smoothly at the peak, then declines. The peak productivity stays at the post-median part. This indicates that the economic productivity increases in the most part of the lifetime, only decreases at the end of the life. 


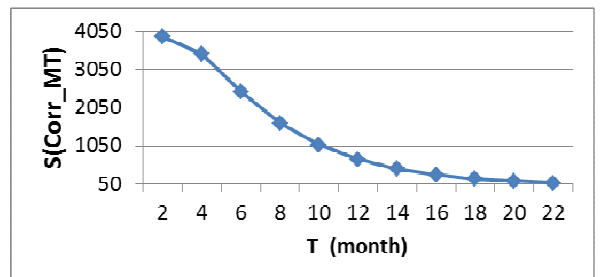

Fig. 1. Without influences of factors, maintenance value changes with maintenance time $T$

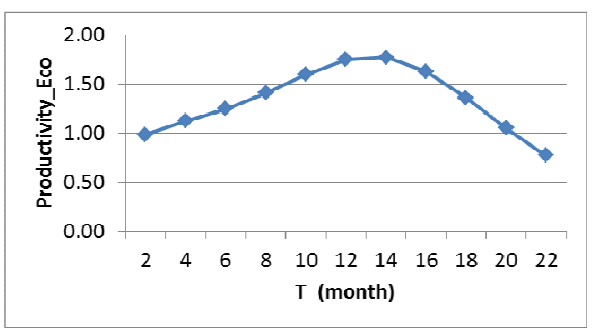

Fig. 2. Without influences of factors, maintenance productivity changes with maintenance time $T$.

In the above analysis, we assume the case that the corrective maintenance is not influenced by factors. As the findings in Table 1, there are two groups of factors that influence the productivity in software maintenance. In the following simulations, we will select a number of influence factors for analysis.

\section{B. Influence factor: the quality of system original program}

The quality of the system original program reflects the quality of development result. It is a factor special for maintenance process. This factor $F_{\text {Qual_prog impacts on } 3}$ parameters in the dynamic economic productivity model of maintenance process: the depreciation rate without maintenance $\alpha_{N o_{-} M T}$, pre-committed fixed cost of maintenance $K_{M T}$, and the rate of uncertainty $\sigma$.

- Analyse the influence $F_{\text {Qual_prog }}$ to depreciation rate

If the quality of the original software program is high, the system should run relatively steady. Higher quality means less bugs, better performance, and more precise design. With good customer operational experience and outstanding presentation, the life of the system is extended and the value of the system increases. The depreciation rate without maintenance (natural depreciation rate) $\alpha_{N o_{-} M T}$ of a system with higher development quality should be greater than that with lower development quality. To model the relationship between $\alpha_{N_{-} M T}$ and the factor of quality of system original program

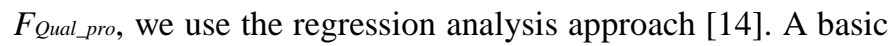
linear regression model is built to specify this relationship as follows:

$$
\alpha_{\text {No_MT }}=\beta_{\alpha 0}+\beta_{\alpha 1} \times F_{\text {Qual_prog }_{\text {pro }}}+\varepsilon_{\alpha} \quad \beta_{\alpha 1}>0
$$

where $\beta_{\alpha 0}$ is the location invariant, $\beta_{\alpha 1}$ is the scale invariant, and $\varepsilon_{\alpha}$ is the error term in the regression model.

- Analyse the influence of $F_{\text {Qual_prog }}$ to fixed cost $K_{M T}$
Meanwhile, high quality of the system original program can save the efforts of maintenance task for maintainers. High quality system needs a few maintenance personnel. Even there are some bugs, high quality program makes it easy for maintainers to read source code and position the problem. Therefore the effort and the cost of maintenance personnel are reduced. The cost of maintenance personnel is a part of precommitted fixed cost $K_{M T}$. So the influence factor of quality of the system original program $F_{\text {Qual_prog }}$ is negatively correlated with $K_{M T}$. We assume that the relation between pre-committed fixed cost $K_{M T}$ and the factor $F_{\text {Qual_prog }}$ can be described by a unary linear regression model:

$$
K_{M T}=\beta_{K 0}+\beta_{K 1} \times F_{\text {Qual }_{-} \text {prog }}+\varepsilon_{K} \quad \beta_{K 1}<0
$$

where $\beta_{K 0}$ is the location invariant, $\beta_{K 0}$ is the scale invariant, and $\varepsilon_{K}$ is the error term of the model.

- Analyse the influence of $F_{\text {Qual_prog }}$ to uncertainty rate $\sigma$

The quality of the system original program is negatively correlated with the rate of uncertainty $\sigma$ too. Systems with low quality programs are always along with troubles and unexpected results. The rate of uncertainty is increased with the decrease of the quality of the system. A basic unary linear regression model is built to describe the relation between $\sigma$ and the factor FQual_prog:

$$
\sigma=\beta_{\sigma 0}+\beta_{\sigma 1} \times F_{\text {Qual_ }_{-} \text {prog }}+\varepsilon_{\sigma} \quad \beta_{\sigma 1}<0
$$

where $\beta_{\sigma 0}$ is the location invariant, $\beta_{\sigma 1}$ is the scale invariant, and $\mathcal{E}_{\sigma}$ is the error term in the regression model.

\section{- Analysis of the influence of $F_{\text {Qual_prog }}$ to productivity}

Now we can analyse the influence of the quality of the system original program $F_{\text {Qual_prog }}$ on the economic productivity Productivity_Eco. In simulation, keep the value of $S_{D e v}, T, r$, and $\alpha_{C_{\text {Orr_MT }}}$ unchanged. We set the value of the new developed system $S_{D e v} 10000$ dollars per month, system maintenance time $T 12$ months, discount rate $r$ at $3 \%$ per month, depreciation rate with corrective maintenance $\alpha_{\text {Corr } M T}$ 0.8. The coefficients of the three regression models in Eq. (9), (10) and (11) are set as below:

$$
\begin{array}{lll}
\beta_{\alpha 0}=0.33 & \beta_{K 0}=-400 & \beta_{\sigma 0}=-0.3 \\
\beta_{\alpha 1}=0.2 & \beta_{K 1}=1400 & \beta_{\sigma 1}=0.55
\end{array}
$$

The standard quality of the system original program $F_{\text {Qual_prog }}$ is assigned to 1 . In the simulation, $F_{\text {Qual_prog }}$ changes from 0.6 to 1.5 with the interval of 0.05 . The quality of the system original program is poor when $F_{\text {Qual_prog }}$ is 0.6 , and the quality is high when $F_{\text {Qual_prog }}$ is 1.5 . The results are demonstrated by Figs 3, 4, 5, and 6.

From the simulation results in Fig 3, 4, 5 and 6, we find out that as the quality of the original system program $F_{\text {Qual_prog }}$ goes up, the unit value of the maintenance $S_{M T}$ declines, the pre-committed fixed cost of maintenance declines, and the rate of uncertainty $\sigma$ declines too. But the economic productivity of maintenance process Productivity_Eco grows up. 


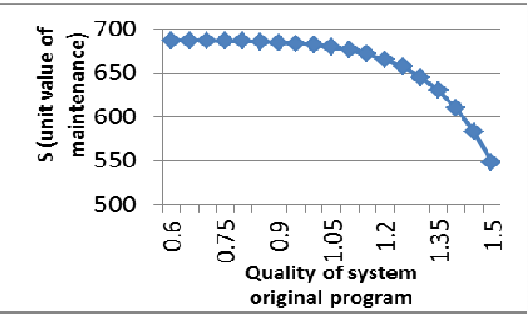

Fig. 3. The unit value of the maintenance in a unit time $S M T$ changes with the factor of the quality of the original program FQual_prog.

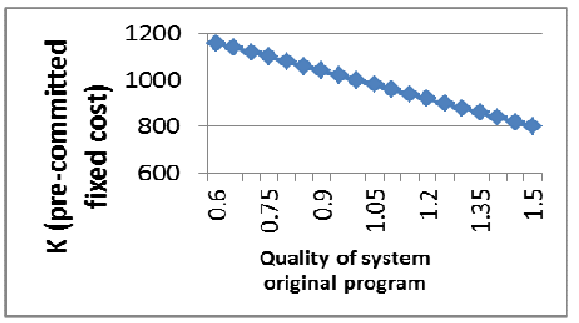

Fig.4. Pre-committed fixed cost of maintenance $K M T$ changes with the factor of the quality of the original program $F$ Qual_prog.

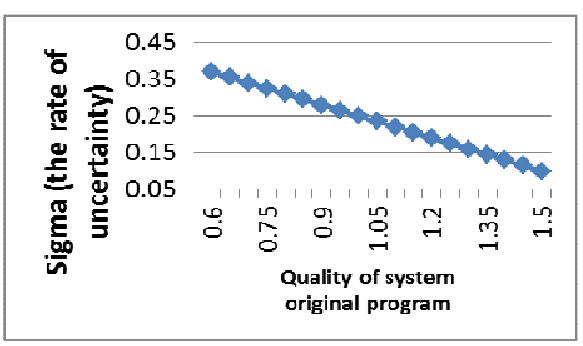

Fig. 5. The rate of uncertainty $\sigma$ changes with the factor of the quality of the original program FQual_prog.

We observed that the unit value of the maintenance $S_{M T}$ slides accelerated as the program quality goes up steady. That means the better the original program is, the much less necessary the corrective maintenance will be, the much less the value of maintenance will be. The ascending velocity of Productivity_Eco becomes bigger and bigger with the steady

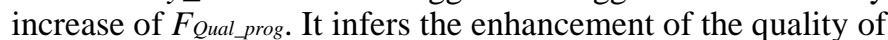
the system original program can earn bigger enhancement of the economic productivity in corrective maintenance.

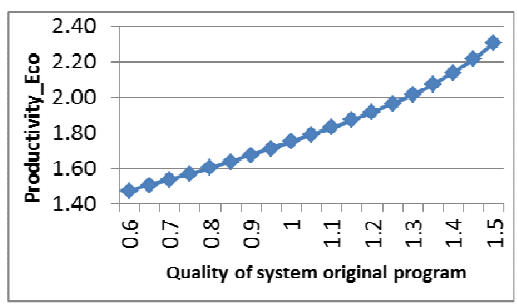

Fig. 6. The relation between the economic productivity Productivity_Eco and facor of the quality of the system original program FQual_prog.

From the result we conclude that to achieve high economic productivity, decrease the total cost of the system (development cost + maintenance cost), it is necessary to improve the quality of the original program. Most effort and time in development process, more enhancement of the program quality will be achieved. Strategies to improve the program quality can be derived as: employing qualified and experienced programmers, designing a detailed requirement statement, and arranging sufficient development time, etc.

In this section, we select one impact factor to analyse the productivity in corrective maintenance. The simulation results for other factors will be presented in future research.

\section{CONCLUSIONS AND FURTHER RESEARCH}

In this paper, we developed an analytical economic model for software maintenance productivity. Impact factors to maintenance process are identified. There are three major maintenance types. Corrective maintenance type is modelled in this paper. Among the impact factors, the quality of the original software program is selected to demonstrate the analysis result in simulation. By using the model simulation, this economic model can be used as a tool to analyse the impact factors and select the optimal parameters to achieve the optimal productivity. In further research, other impact factors will be analysed. Adaptive maintenance and perfective maintenance will be modelled.

\section{REFERENCES}

[1] ISO/IEC/IEEE 24765:2010(E) "Systems and software engineering Vocabulary"

[2] Nguyen, Vu, Barry Boehm, and Phongphan Danphitsanuphan."A Controlled Experiment in Assessing and Estimating Software Maintenance Tasks." Information and software technology 53,6 (2011): 682-91.

[3] Boehm B.W. Understanding and controlling software costs, IEEE Trans. Software Engineering. Vol. 14, No. 10. pp.1462-1477, (1988).

[4] Lientz, Bennet P., E. Burton Swanson, and Gail E. Tompkins. "Characteristics of Application Software Maintenance." Communications of the ACM 21,6 (1978): 466-71

[5] Boehm, Barry W. "Software Engineering Economics." Prentice-Hall Advances in Computing Science and Technology Series, Englewood Cliffs: Prentice-Hall, 1981

[6] Albrecht, A. J., and J. J. Gaffney. "Software Function, Source Lines of Code, and Development Effort Prediction: A Software Science Validation." IEEE Transactions On Software Engineering SE-9,6 (1983):639-48.

[7] Liu L., Kong X., and Chen J. How Project Duration, Upfront Costs And Uncertainty Interact And Impact On Software Development Productivity? A Simulation Approach, Int. J. of Agile Systems and Management, In Press

[8] Swanson, E. Burton. "The dimensions of maintenance." Proceedings of the 2nd international conference on Software engineering. IEEE Computer Society Press, 1976.

[9] Jorgensen, Magne. "Experience with the accuracy of software maintenance task effort prediction models." Software Engineering, IEEE Transactions on 21.8 (1995): 674-681.

[10] Basili, Victor, et al. "Understanding and predicting the process of software maintenance release." Proceedings of the 18th international conference on Software engineering. IEEE Computer Society, 1996.

[11] IEEE (1992) IEEE Standard for Software Productivity Metrics 1045

[12] Chen, J. (2012) The Nature of Discounting, Structural Change and Economic Dynamics, Vol. 23, p. 313-324. 2012

[13] Chen, J. (2005) THE PHYSICAL FOUNDATION OF ECONOMICS, An Analytical Thermodynamic Theory, World Scientific Publishing Co. 2005.

[14] Frees, Edward W "Regression modeling with actuarial and financial applications", Cambridge University Press, 2010. 